\title{
AVOIDANCE OF ARTIFACTS IN HARMONIC BALANCE SOLUTIONS FOR NONLINEAR DYNAMICAL SYSTEMS
}

\author{
LUKAS LENTZ, UTZ VON WAGNER \\ Technische Universität Berlin, Chair of Mechatronics and Machine Dynamics, Berlin, Germany \\ e-mail:lukas.lentz@tu-berlin.de; utz.vonwagner@tu-berlin.de
}

\begin{abstract}
Although nonlinear mechanical systems have been the topic of numerous investigations during the last decades, the research on suitable analysis methods is still ongoing. One method that is commonly known and still sees a lot of interest is the Harmonic Balance method. In the basic version of this method, only one harmonic is used to approximate a periodic solution, which allows for fairly easy application. A drawback is that this approach may lead to solutions that are inaccurate or even artifacts which are solutions that possess no physical relevance. In this article, it is demonstrated how an error criterion can be used to access the accuraccy of solutions and how artifacts can be indentified based on this assessment. Subsequently, stability analysis is performed for solutions that possess small errors. The method is applied to an asymmetric Duffing oscillator as well as to a system that consists of two linearly coupled Duffing oscillators.

The authors gave a corresponding presentation of their work at PCM-CCM Kraków 2019.

Keywords: nonlinear systems, harmonic balance, artifacts, Duffing oscillators
\end{abstract}

\section{Introduction}

The Duffing oscillator, named after German engineer Georg Duffing (1861-1944), is one of the classic academic examples for nonlinear oscillators (Nayfeh and Mook, 1979). In addition to, it is an academic value seeing a lot of attention due to it is capability to model various systems, ranging from neuronal groups in the human brain (Srebro, 1995) to bistable beam systems that are frequently used as a base structure in energy harvesting applications (Erturk and Inman, 2011).

A comprehensive summary of the recent research activities on the Duffing oscillator can be found in the book (Kovacic and Brennan, 2011) edited by Kovacic et al., where also an English translation of the original article from Duffing (1918), that was written in German, can be found. In the original article, a single degree of freedom oscillator with quadratic and cubic stiffness terms is considered, what is in contrast to the actual use of the name Duffing-oscillator, which is typically associated with a purely cubic nonlinearity.

In this paper, we consider the classical system, including quadratic and cubic terms in the nonlinearity as well as a system consisting of two linearly coupled Duffing oscillators with solely cubic terms in the nonlinearity. For both systems, the response to a harmonic excitation is computed using an extended form of the Harmonic Balance method in which an error criterion is used to assess the quality of computed solutions and rule out artifacts which are solutions that possess no physical relevance.

The Harmonic Balance method is a special case of the Galerkin method in which a series of harmonic functions is used for construction of an approximation for the solution of a nonlinear dynamical system. A comprehensive mathematical description of the method can be found in (Urabe, 1965) including a proof for the existence of periodic solutions and the convergence of the 
approximations against the unknown exact solutions. Furthermore, an estimate for the upper bound of the error in the fulfillment of the differential equation and the distance to the unknown exact solution is given therein. Examples for the numerical application of the method are given in (Urabe and Reiter, 1966) where the method is used for the analysis of various one dimensional nonlinear systems. Further details on the theory are given in (Stokes, 1972) and more lately in (García-Saldaña and Gasull, 2013), where the aim is to derive general statements about the accuracy of the approximations and an improved measure for the distance to unknown exact solutions. General comments on the application of such error-estimates can be found in (Ferri and Leamy, 2009).

Nowadays, the Harmonic Balance method is one of the most-used methods in nonlinear dynamics, besides other semi-analytical approximation methods like the perturbation analysis (e.g. Lindstedt-Poincaré) or the method of Multiple Time scales. If the used approximation is limited to a single harmonic, the Harmonic Balance method is, in general, easy to handle and the resulting solution often provides a good insight in the dynamical behavior of the system under consideration. However, if the exact solution of the system contains more than one harmonic component, the computed approximations may deviate substantially from the excact solutions. Furthermore, it is possible that artifacts, which are sometimes also called spurious- or non-physical solutions, occur as it was demonstrated e.g. in (von Wagner and Lentz, 2016, 2018). A more detailed explanation what we mean by "artifacts" will be given in the next Section.

Although the theoretical body of research on the Galerkin-method is large, the research on such artifacts is remarkably small. To our best knowledge, the only article by an other author, where artifacts in conjunction with the Harmonic Balance method are mentioned, is (Liu et al., 2006). In this article, the artifact solutions are named non-physical solutions and the non-convergence of the coefficients in the approximation is used to verify this statement. Although this approach is valid, it has the disadvantage that it requires a large number of solutions to assess the state of the convergence. Therefore, it seems to be favourable to use the approach that is presented in (von Wagner and Lentz, 2016) where an error criterion is used to identify artifacts in the solution of a softening Duffing oscillator. As it is shown there, this approach can be used to identify artifacts at a relatively low computational cost. In further publications, the proposed approach is applied for the analysis of more complex systems, e.g. a piezo related system (von Wagner and Lentz, 2018) and in (von Wagner and Lentz, 2019) it is shown that the proposed approach provides qualitatively similar results as the assessment of the convergence of coefficients for the therein considered examples.

\section{Description of the Harmonic Balance method}

In this Section, a general description of the Harmonic Balance method is given following (Urabe, 1965). For the sake of simplicity a single degree of freedom system is considered, which does not pose a strong restriction since the generalization to a system with multiple degrees of freedom is straight forward. The system is given by the equation of motion

$$
x^{\prime \prime}(\tau)+g\left(x^{\prime}(\tau), x(\tau), \tau\right)=0
$$

wherein $x$ is the generalized coordinate of the system, $\tau$ a dimensionless time, $(\cdot)^{\prime}$ describes the derivative with respect to $\tau$ and $g$ is a polynomial type nonlinearity in $x$ as well as $x^{\prime}$ and is periodic with respect to $\tau$.

The objective is to construct regular (i.e. non-chaotic) solutions $x$ that are also periodic in $\tau$ with the same period T. According to (Urabe, 1965), nonlinear system (2.1) possesses one or multiple periodic solutions that can be approximated by the harmonic series 


$$
x(\tau)=a_{0}+\sum_{i=1}^{n}\left[a_{i} \cos (i \eta \tau)+b_{i} \sin (i \eta \tau)\right]
$$

with unknown coefficients $a_{i}, b_{i}$. Herein $\eta$ denotes a dimensionsless circular frequency which often represents the excitation frequency and is related to the period by $T=2 \pi / \eta$.

The most basic version of the Harmonic Balance method, which will be referred to as the monofrequent Harmonic Balance method, is obtained if the series is limited to a single harmonic (i.e. $n=1$ ) that may be supplemented by an additional constant term. If multiple harmonic functions are used (i.e. $n \geqslant 2$ ), the method will be referred to as the higher order Harmonic Balance method.

Introducing ansatz (2.2) into differential equation (2.1), and expanding the result in the respective harmonics, yields

$$
\frac{\widetilde{a}_{0}}{2}+\sum_{i=1}^{n}\left[\widetilde{a}_{i} \cos (i \eta \tau)+\widetilde{b}_{i} \sin (i \eta \tau)\right]=-\sum_{i=n+1}^{m}\left[\widetilde{a}_{i} \cos (i \eta \tau)+\widetilde{b}_{i} \sin (i \eta \tau)\right]
$$

where $m>n$ holds, if the system is nonlinear. If, for example, $g$ is of the third order, as in the case of the Duffing oscillator, we have $m=3 n$. The new coefficients $\widetilde{a}_{i}, \widetilde{b}_{i}$ are given by the integrals

$$
\begin{aligned}
& \widetilde{a}_{i}=\frac{2}{T} \int_{0}^{T}\left[x^{\prime \prime}(\tau)+g\left(x^{\prime}(\tau), x(\tau), \tau\right)\right] \cos (i \eta \tau) d \tau \\
& \widetilde{b}_{i}=\frac{2}{T} \int_{0}^{T}\left[x^{\prime \prime}(\tau)+g\left(x^{\prime}(\tau), x(\tau), \tau\right)\right] \sin (i \eta \tau) d \tau
\end{aligned}
$$

which are nonlinear functions of the original coefficients in (2.2). Since the harmonics in (2.3) are linearly independent, each of the new coefficents in (2.3) must be equal to zero, which yields a system of $2 m+1$ nonlinear algebraic equations. Because the number of original coefficients in (2.2), which are the independent variables in those equations, is $2 n+1$ with $m>n$, the system is overdetermined. Following the Harmonic Balance method for calculation of these $2 n+1$ unknown coefficients, higher order terms (i.e. terms with higher order than $n$ ) are neglected, and the solution is computed from the first $2 n+1$ equations.

At this point, it should be pointed out that the remaining $2(m-n)$ equations, which are neglected in the Harmonic Balance method, would also be equal to zero if the computed approximation would represent an exact solution. Since this is generally not the case, the values of those neglected equations describe the discrepancy in the fulfillment of the differential equation and can therefore be used to define a measure for the error that is made by this approximation.

In (Urabe, 1965) this error is defined as

$$
e_{\text {Urabe }}=\sum_{i=n+1}^{m} \sqrt{\widetilde{a}_{i}^{2}+\widetilde{b}_{i}^{2}}
$$

which is only an upper bound for the error, since there might be a phase shift between the respective harmonics that are contained in the error. Therefore, it is reasonable following (von Wagner and Lentz, 2019) to expect a closer assessment of the error by

$$
\widetilde{e}=\max _{\tau}\left\{\sum_{i=n+1}^{m}\left[\widetilde{a}_{i} \cos (i \eta \tau)+\widetilde{b}_{i} \sin (i \eta \tau)\right]\right\}
$$


To further reduce the influence of the absolute magnitude of the coefficients as also in (von Wagner and Lentz, 2019), the error is divided by the absolute maximum of the corresponding solution

$$
x_{\max }=\max _{\tau}\left\{\frac{\widetilde{a}_{0}}{2}+\sum_{i=1}^{n}\left[\widetilde{a}_{i} \cos (i \eta \tau)+\widetilde{b}_{i} \sin (i \eta \tau)\right]\right\}
$$

which leads to the relative error

$$
e=\frac{\widetilde{e}}{x_{\max }}
$$

that will be used for the assessment of the accuracy of an approximation in the following, where the value is usually given in percentage. For the application, it is important to note that there is no general rule how to decide which error value is admissible. From our experience, this problem can be overcome if the change that occurs in the error values when the number of harmonics in the ansatzfunction is increased, is evaluated instead. To be more specific, we compute approximations for an increasing number of harmonics in the ansatzfunction and look for approximations where the error values do not change significantly when the number of harmonics is increased. If such solutions are found, they are considered to be artifacts.

In our understanding, artifacts are solutions which solve the system of nonlinear equations that occur throughout the approximation process, but are no solutions of the underlying differential equation.

\section{Asymmetric Duffing oscillator}

In two former publications of the authors (von Wagner and Lentz, 2016, 2018) the described method was used to analyze a Duffing oscillator with a purely cubic nonlinearity. Therein, it is shown how the application of the monofrequent Harmonic Balance method leads to artifact solutions in the considered example in which a negative cubic nonlinearity (softening behavior) was used.

In (von Wagner and Lentz, 2019), the parameter set from van Dooren (1988) was used to demonstrate that those large amplitude solutions were not always artifacts. Using this specific example, it was shown how the initially large relative errors decreased when the number of harmonics was increased, i.e. in (von Wagner and Lentz, 2019) $n=1,3,15,33,41,49$, which indicated that the corresponding solutions were no artificats but valid solutions. For the stable part of those solutions it was possible to confirm these results by means of numerical integrations.

In the present article, we supplement the prior investigations by the analysis of a harmonically excited Duffing oscillator with both quadratic and cubic nonlinearity

$$
x^{\prime \prime}(\tau)+\xi x^{\prime}(\tau)+\alpha x(\tau)+\beta x^{2}(\tau)+\gamma x^{3}(\tau)=f \cos (\eta \tau)
$$

where $\xi$ denotes the damping coefficient, $\alpha, \beta$ and $\gamma$ denote the coefficients of the linear, quadratic and cubic stiffness, $f$ is the amplitude of the external excitation and all other denominations follow those in the previous Section. The parameter values that are used for the following investigation can be found in Table 1.

Table 1. Parameter values for Fig. 1

\begin{tabular}{|c|c|c|c|c|c|}
\hline symbol & $\xi$ & $\alpha$ & $\beta$ & $\gamma$ & $f$ \\
\hline \hline value & 0.11 & 1 & 0.08 & -0.3 & 0.1 \\
\hline
\end{tabular}



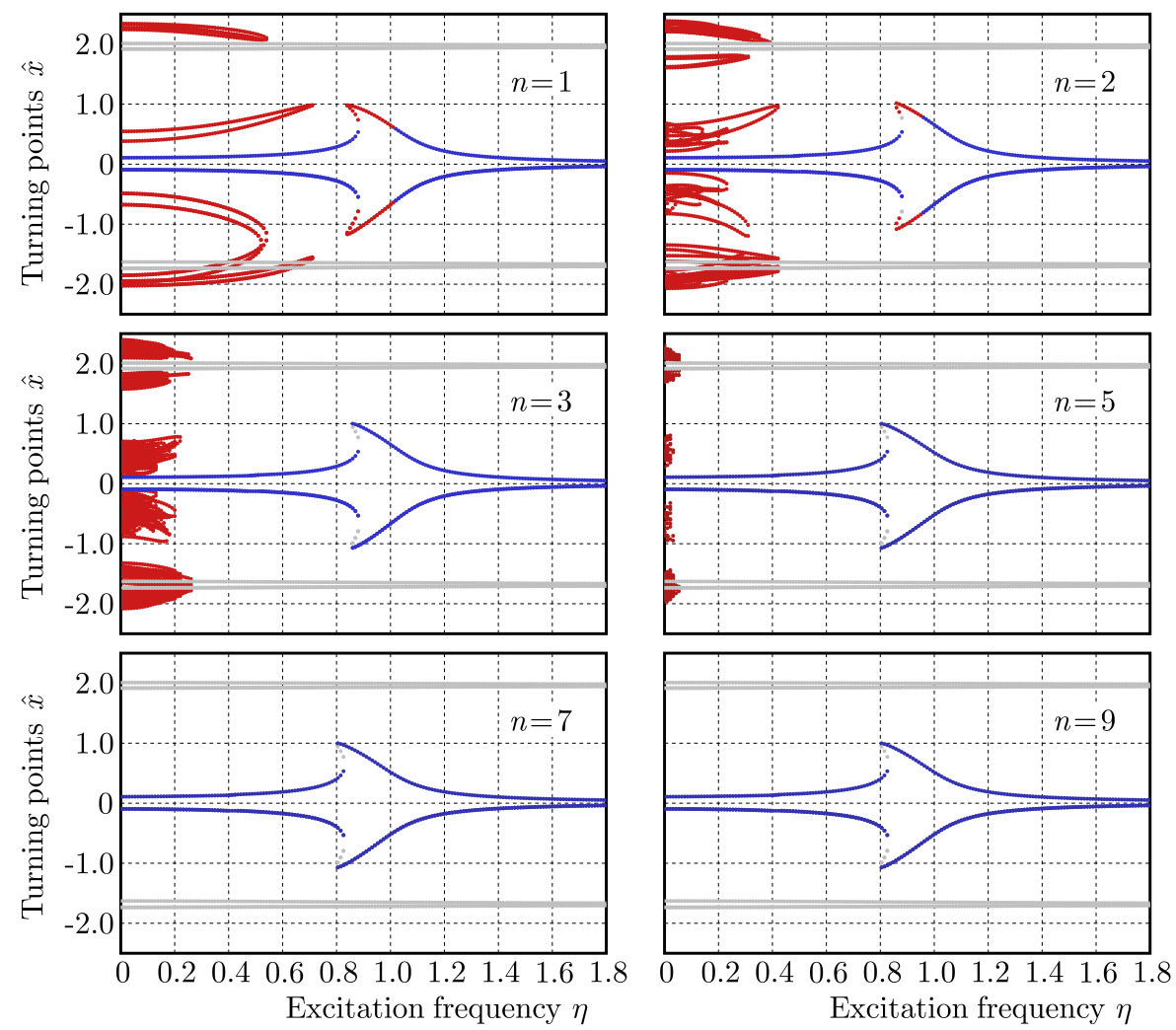

Fig. 1. Harmonic Balance solutions of equation (3.1) with the increasing ansatz order $n$ according to equation (2.2). Solutions plotted in red have the relative error $e$ according to (2.8) larger than 1\%, solutions plotted in blue have the relative error $e$ lower than $1 \%$ and are asymptotically stable and solutions that are plotted in gray also have the relative error $e$ lower than $1 \%$ but are unstable

The analysis follows the description above for $n=1,2,3,5,7,9$. The computed results are gathered in Fig. 1 where the turning points $\widehat{x}$ (i.e. all points $x$ where the velocity vanishes) are plotted in dependence of the excitation frequency $\eta$.

For each set of solutions that is computed for a specific value of $n$, the relative error is determined and a threshold value of $1 \%$ is used for the classification of the solutions. If the relative error is above the threshold value, the corresponding solution is plotted in red and no further assessment of the solution is done. However, if the relative error is below the threshold value, the stability of the corresponding solution is analysed, using Floquet's theory. On that basis solutions are plotted in blue if they have a small relative error and are asymptotically stable and in gray if they have a small relativ error and are unstable.

The analysis of the results starts with the case $n=1$ (see uper left corner in Fig. 1). As it can be seen only those solutions which oscillate with a small amplitude have a relative error below the threshold value. Those solutions are the well known stable solutions that oscillate around the origin (blue) and the unstable solutions described in (von Wagner and Lentz, 2016, 2018) that oscillate around the non zero equilibrium positions (gray). The remaining solutions, which all possess a large relative error, can be divided into two groups. The first there are solutions that occur in the frequency range $0 \leqslant \eta \leqslant 0.8$ which oscillate with a large amplitude and look similar to the solutions that were identified as artifacts in the case of the softening Duffing oscillator in (von Wagner and Lentz, 2016, 2018). Secondly, there are solutions that occur in the frequency range $0.8<\eta<1.5$ which are a part of the solution branch that consists of stable solutions with small amplitudes. Based on a general knowledge on nonlinear oscillations, it is reasonable to expect that those solutions are no artifacts. A study of the results with an increased number of harmonics confirms this. For $n \geqslant 3$, the relative error of the latter described solutions decreases below the threshold, and the expected stability behavior is found. 
If the first group of solutions is considered, the result is significantly different. As it can be seen, the relative error of the solutions remains large despite the increase in the number of harmonics. Furthermore, the shape of solutions changes drastically from $n=1$ to $n=3$ and new solutions which also have a large relative error are found for $n=2$ and $n=3$. Finally, all those solutions disappear for $n>5$ which shows that they do not represent a converging approximation. This leads to the conclusion that all the solutions from this group are artifacts.

\section{Coupled Duffing oscillators}

As a second example the system of two coupled Duffing oscillators, that was investigated in (Kozłowski et al., 1995) by means of numerical integrations, is analyzed. This system is given by

$$
\begin{aligned}
& x^{\prime \prime}+0.1 x^{\prime}+x+5(x-y)+x^{3}=f \cos (\eta \tau) \\
& y^{\prime \prime}+0.1 y^{\prime}+y+5(y-x)+y^{3}=0
\end{aligned}
$$

wherein $x$ and $y$ denote the two generalized coordinates. The coupling only takes place via the linear terms, and in equation (4.1) 1 there is a harmonic excitation, while the vibration in $y$ is only excited by the coupling of the two equations. The parameter values for the linear damping, and the linear as well as nonlinear stiffness coefficients are the same values that were used in (Kozłowski et al., 1995).

The extended ansatz (2.2) for the two coordinates is

$$
\begin{aligned}
& x(\tau)=a_{x, 0}+\sum_{i=1}^{n}\left[a_{x, i} \cos (i \eta \tau)+b_{x, i} \sin (i \eta \tau)\right] \\
& y(\tau)=a_{y, 0}+\sum_{i=1}^{n}\left[a_{y, i} \cos (i \eta \tau)+b_{y, i} \sin (i \eta \tau)\right]
\end{aligned}
$$

and the following Harmonic Balance analysis follows the description that was given for the one degree of freedom system.

Figure 2 shows the results from an application of the monofrequent Harmonic Balance $n=1$ and varying values for the excitation amplitude $f$. As could be expected, due to the stiffening characteristics, the resonance peaks bow to the right. Beside this, depending on the excitation amplitude, a coexistence of the solutions can be observed for wide ranges of the excitation frequency $\eta$. The question in the following will be whether the solutions are artifacts or valid physical solutions. It should be mentioned that in general the chosen parameters are not resulting in small nonlinearities which would make the application of other semi-analytic procedures as the Lindstedt-Poincaré method or the method of Multiple Time scales difficult.

In the following, the case $f=10.0$ is discussed in more detail. Figure 3 demonstrates again that the impression, which is given by the monofrequent Harmonic Balance with $n=1$, may be delusive especially for low values of the excitation frequency $\eta$. For $n=1$, almost all solutions have relative errors larger than $1 \%$. For an increasing $n$, several of the solutions obtained for $n=1$ are confirmed by keeping their basic shape together with a decrease in the relative errors although several of them are identified as unstable. For low excitation frequencies $\eta \leqslant 1.5$,the figure changes for large $n$ compared to $n=1$. There are even $\eta$ where no stable solution is found by the Harmonic Balance method following ansatz (4.2). One possible reason is that stationary solutions exist at these $\eta$, but cannot be represented by ansatz (4.2). For example, these can be solutions with period multiplications which would require not only the use of multiples i $\eta$ with natural numbers $i$ as multiples of the excitation freqency in the ansatz, but also of fractions of $\eta$. 

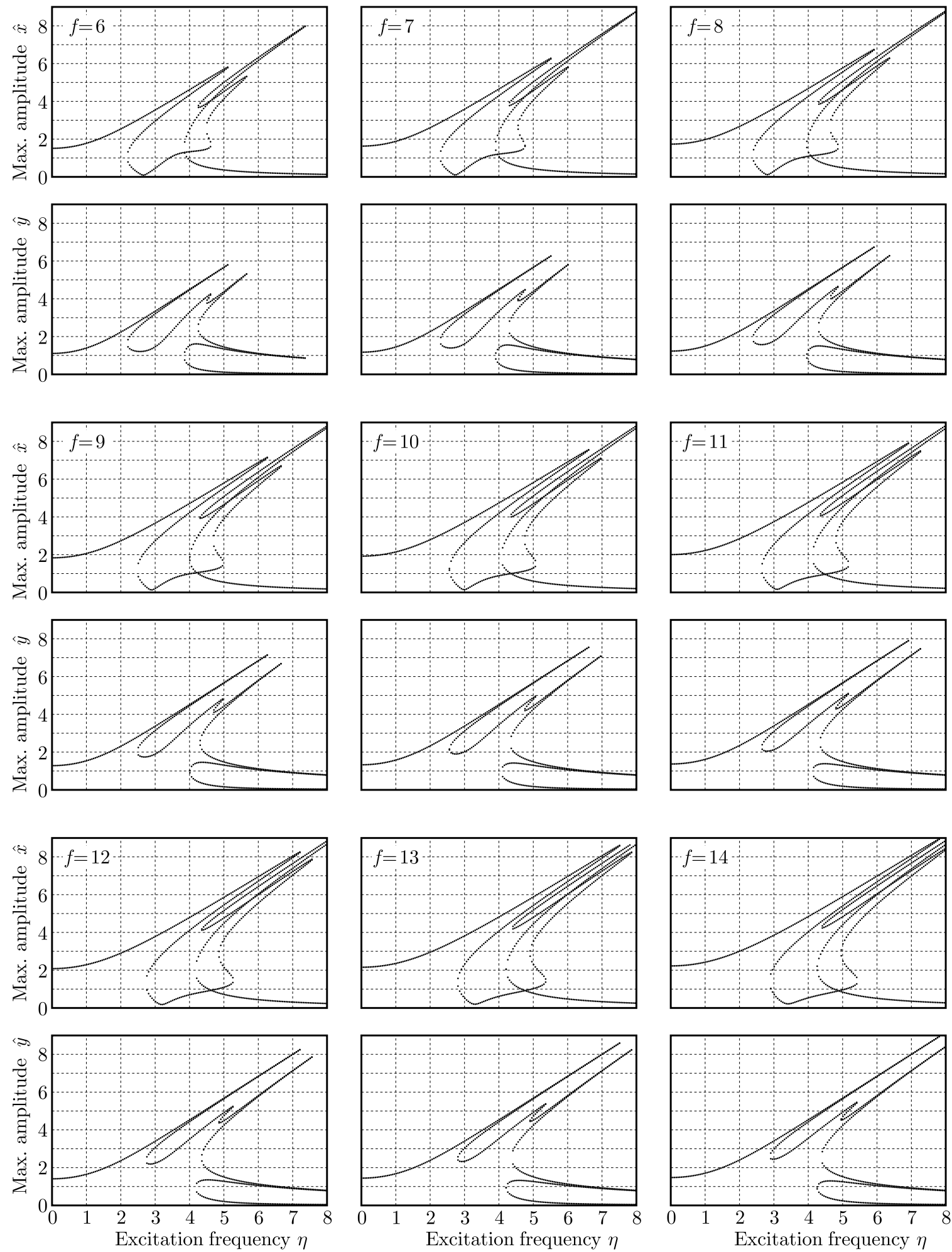

Fig. 2. Solutions of the monofrequent Harmonic Balance analysis of equations (4.1) with ansatz (4.2) for varying $f$

Another possibility could be that no stationary but only chaotic solutions exist which cannot be represented by a harmonic expansion. Answering this question requires modified expansions or calculation of solutions via numerical integration, which shall be a part of the future work. 

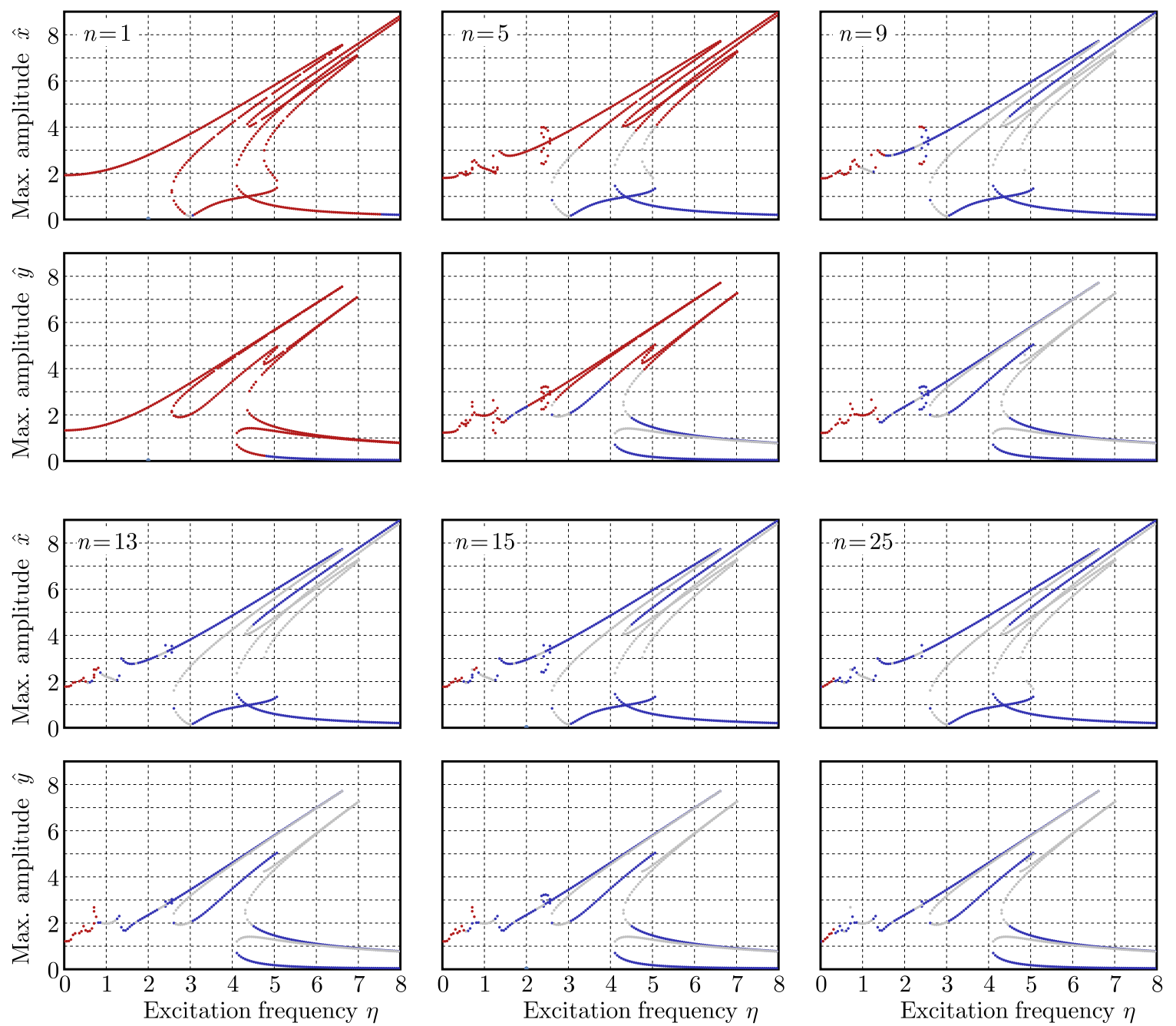

Fig. 3. Harmonic Balance solutions of equations (4.1) with ansatz (4.2) in the case of increasing $n$ for $f=10.0$. Solutions plotted in red have the relative error $e$ according to $(2.8)$ larger than $1 \%$, solutions plotted in blue have the relative error $e$ lower than $1 \%$ and are asymptotically stable, solutions plotted in gray are solutions with the relative error $e$ lower than $1 \%$ but unstable

Both examples, the quadratic-cubic single degree of freedom oscillator as well the two coupled cubic oscillators, demonstrate that the application of the most basic version of the Harmonic Balance method with $n=1$, i.e. the monofrequent Harmonic Balance method may in general result, depending on the specific example and parameters, in a wrong impression of the variety of solutions, i.e. the occurence of artifacts. To avoid such artifacts, which means to avoid the acceptance of any Harmonic Balance solutions as physical solutions without additional proof, higher ansatz orders $n$ should be applied in conjunction with the described error criterion.

\section{Conclusions}

In this paper, two examples of nonlinear oscillators were analyzed by the Harmonic Balance method. This method includes application of an error criterion, consideration of higher ansatz orders as well as stability analysis of small error solutions based on Floquet's theory. For both analyzed examples it could be shown that the frequently used monofrequent Harmonic Balance method (i.e. $n=1$ ) may lead to erroneous results due to the occurence of artitacts. As artifacts we have denoted solutions that are solutions of the system of nonlinear algebraic equations resul- 
ting from the Harmonic Balance method, but are no (approximate) solutions of the underlying differential equations. Those artifacts are identified by consideration of the relative error for which a threshold value of $1 \%$ was used in our examples. If the relative error remains above that threshold, even for an increased ansatz order $n$ or if the solution dissapears, it is considered to be an artifact. The two examples in the present paper, the first one a Duffing oscillator with quadratic and cubic nonlinearity, the second one a system of two coupled Duffing oscillators, showed various cases of the occurence of artifacts. As it has been shown, the proposed method provides a reliable method for detection of artifacts and is comparably easy to apply. Based on the proposed method it is possible to remove the artifacts from the computed solutions and, therefore, avoid their further usage.

Acknowledgement

This work is funded by the Deutsche Forschungsgemeinschaft (DFG, German Research Foundation) with grant WA 1427/30-1.

\section{References}

1. Duffing G., 1918, Forced Oscillations with Variable Natural Frequency and their Technical Significance (in German), Vieweg, Braunschschweig

2. ERturk A., Inman D.J., 2011, Broadband piezoelectric power generation on high-energy orbits of the bistable Duffing oscillator with electromechanical coupling, Journal of Sound and Vibration, 330, 2339-2353

3. Ferri A., LeAmy M., 2009, Error estimates for Harmonic-Balance solutions of nonlinear dynamical systems, 50th AIAA/ASME/ASCE/AHS/ASC Structures, Structural Dynamics, and Materials Conference, DOI: 10.2514/6.2009-2667

4. García-Saldaña J.D., Gasull A., 2013, A theoretical basis for the Harmonic Balance Method, Journal of Differential Equations, 254, 67-80

5. Kovacic I., Brennan M.J., 2011, The Duffing Equation, John Wiley \& Sons, New York

6. Kozlowski J., Parlitz U., Lauterborn W., 1995, Bifurcation analysis of two coupled periodically driven Duffing oscillators, Physical Review E, 51, 1861-1867

7. Liu L., Thomas J.P., Dowell E.H., Attar P.J., Hall K.C., 2006, A comparison of classical and high dimensional harmonic balance approaches for a Duffing oscillator, Journal of Computational Physics, 215, 298-320

8. NaYfen A.H., Mook D., 1979, Nonlinear Oscillations, John Wiley \& Sons, New York

9. Srebro R., 1995, The Duffing oscillator: a model for the dynamics of the neuronal groups comprising the transient evoked potential, Electroencephalography and Clinical Neurophysiology / Evoked Potentials Section, 96, 561-573

10. Urabe M., 1965, Galerkin's procedure for nonlinear periodic systems, Archive for Rational Mechanics and Analysis, 20, 120-152

11. Urabe M., Reiter A., 1966, Numerical computation of nonlinear forced oscillations by Galerkin's procedure, Journal of Mathematical Analysis and Applications, 14, 107-140

12. Stokes A., 1972, On the approximation of nonlinear oscillations, Journal of Differential Equations, 12, $535-558$

13. VAn Dooren R., 1988, On the transition from regular to chaotic behaviour in the Duffing oscillator, Journal of Sound and Vibration, 123, 327-339

14. Von Wagner U., Lentz L., 2016, On some aspects of the dynamic behavior of the softening Duffing oscillator under harmonic excitation, Archive of Applied Mechanics, 8, 1383-1390 
15. Von Wagner U., Lentz L., 2018, On artifact solutions of semi-analytic methods in non-linear dynamics, Archive of Applied Mechanics, 88, 1713-1724

16. Von Wagner U., Lentz L., 2019, On the detection of artifacts in Harmonic Balance solutions of nonlinear oscillators, Applied Mathematical Modelling, 65, 408-414

Manuscript received December 20, 2019; accepted for print January 24, 2020 